\title{
Anti-CD20-atezolizumab-polatuzumab vedotin in relapsed/refractory follicular and diffuse large B-cell lymphoma
}

\author{
Max S. Topp ${ }^{1}$ (1) $\cdot$ Herbert Eradat $^{2} \cdot$ Axel Florschütz $^{3} \cdot$ Andreas Hochhaus $^{4} \cdot$ Tomasz Wrobel $^{5} \cdot$ Jan Walewski $^{6}$. \\ Wanda Knopinska-Posluszny ${ }^{7} \cdot$ Abraham S. Kanate $^{8} \cdot$ Ewa Lech-Maranda $^{9} \cdot$ Uta Brunnberg $^{10} \cdot$ Surya Chitra $^{11}$. \\ Tina G. Nielsen ${ }^{12} \cdot$ Gila Sellam $^{12} \cdot$ Mahesh Shivhare $^{13} \cdot$ Izidore S. Lossos $^{14}$
}

Received: 31 March 2021 / Accepted: 30 October 2021 / Published online: 18 February 2022

(c) The Author(s) 2022

\begin{abstract}
Purpose New therapies are needed for relapsed/refractory (R/R) B-cell non-Hodgkin lymphoma. This phase 1b, open-label trial evaluated two anti-CD20-based triplet combinations.

Methods Patients with R/R follicular lymphoma (FL; $n=13$ ) were treated with obinutuzumab, atezolizumab, and polatuzumab vedotin (G-atezo-pola; $1.4 \mathrm{mg} / \mathrm{kg} / 1.8 \mathrm{mg} / \mathrm{kg}$ ) and patients with R/R diffuse large B-cell lymphoma (DLBCL; $n=23)$ received rituximab (R)-atezo-pola. The primary efficacy endpoint was complete response (CR) at end of induction (EOI) by PET-CT (investigator assessed; modified Lugano 2014 criteria). Safety endpoints were also assessed.

Results 13 FL patients were treated and evaluable for safety; 2/23 DLBCL patients did not receive treatment and were not included in the safety population. Median observation time was 23.3 and 5.7 months in the FL and DLBCL cohorts, respectively. At EOI, CR rates in FL patients treated with G-atezo-pola at pola doses of $1.4 \mathrm{mg} / \mathrm{kg}(N=3)$ and $1.8 \mathrm{mg} / \mathrm{kg}$ $(N=7)$ were $33 \%$ and $14 \%$, respectively. In DLBCL patients receiving R-atezo-pola, the CR rate at EOI was $13 \%$. In the FL cohort, $62 \%$ of patients experienced a grade 3-5 adverse event (AE; including two deaths) and $31 \%$ developed a serious AE (SAE). In DLBCL patients, R-atezo-pola was associated with a lower incidence of grade 3-5 AEs (24\%; one death) and SAEs (10\%). In both cohorts, the most common grade 3-5 AEs were hematologic toxicities.

Conclusion Based on these safety issues, considered as related specifically to G-atezo-pola, and limited efficacy, no further development of either combination is planned.
\end{abstract}

Trial registration NCT02729896; Date of registration: April 6, 2016.

Keywords B-cell non-Hodgkin lymphoma · Diffuse large B-cell lymphoma · Follicular lymphoma · Immunotherapy · Relapsed/refractory

Max S. Topp

topp_m@ukw.de

1 Medizinische Klinik und Poliklinik II, Universitätsklinikum Würzburg, Anstalt des öffentlichen Rechts, Josef-Schneider-Straße 2, 97080 Würzburg, Germany

2 Division of Hematology-Oncology, David Geffen School of Medicine at UCLA, Los Angeles, CA, USA

3 Städtisches Klinikum Dessau, Dessau-Roßlau, Germany

4 Klinik für Innere Medizin II, Universitätsklinikum Jena, Jena, Germany

5 Department of Hematology, Wrocław Medical University, Wrocław, Poland

6 Narodowy Instytut Onkologii im, Marii SkłodowskiejCurie - Panstwowy Instytut Badawczy, Warsaw, Poland
7 Uniwersytet Warmińsko-Mazurski, Olsztynie, Poland

8 West Virginia University Cancer Institute, Morgantown, WV, USA

9 Institute of Hematology and Transfusion Medicine, Warsaw, Poland

10 Universitätsklinikum Frankfurt, Frankfurt, Germany

11 Genentech, Inc., South San Francisco, CA, USA

12 F. Hoffmann-La Roche Ltd, Basel, Switzerland

13 Roche Products Limited, Welwyn Garden City, UK

14 Sylvester Comprehensive Cancer Center, University of Miami, Miami, FL, USA 


\section{Introduction}

Immunotherapy is an emerging approach for the treatment of relapsed/refractory (R/R) B-cell nonHodgkin lymphoma, for which, there is an unmet need for new therapies, including the use of anti-programmed death-1 (anti-PD-1) therapy. Previous studies in B-cell malignancies have demonstrated the potential of combining atezolizumab (anti-PD-ligand 1 [antiPD-L1] antibody) (US FDA 2019; TECENTRIQ ${ }^{\circledR} 2019$ ) or polatuzumab vedotin (pola; anti-CD79b antibodydrug conjugate) (US FDA 2019) with anti-CD20-based therapies, with encouraging response rates (US FDA 2019; TECENTRIQ $^{\circledR}$ 2019; Morschhauser et al. 2019; Phillips et al. 2016; Palomba et al. 2017). Data from the phase $1 \mathrm{~b} / 2$ GO29365 study supported Food and Drug Administration (FDA)-accelerated approval of the first triplet combination therapy in $\mathrm{R} / \mathrm{R}$ diffuse large B-cell lymphoma (DLBCL), incorporating the anti-CD20 antibody, rituximab (R), with pola and bendamustine. Two phase 3 trials, AUGMENT and MAGNIFY, provided evidence for FDA approval of lenalidomide in combination with $\mathrm{R}$ for $\mathrm{R} / \mathrm{R}$ follicular lymphoma (FL) and marginal zone lymphoma. The proven efficacy of anti-CD20 antibody-based therapy supports further investigation into new combinations (Sehn et al. 2016; Ren et al. 2015; Gao et al. 2010). We present the primary analysis of a phase $1 \mathrm{~b}$, open-label multicenter trial of two anti-CD20-based triplet combinations in R/R FL and DLBCL (BO29561; NCT02729896), where recruitment was stopped due to safety concerns. Patients with R/R FL were treated with obinutuzumab (G), atezolizumab (atezo), and pola (G-atezo-pola); patients with R/R DLBCL received R-atezo-pola.

\section{Methods}

\section{Patients, treatment and endpoints}

Eligible patients had previously received $\geq 1$ prior chemoimmunotherapy regimen, including an anti-CD20 antibody (see Online Supplemental Data for eligibility criteria). FL patients were first enrolled in a doseescalation phase to establish the recommended phase 2 dose (RP2D) of pola within the G-atezo-pola regimen. During dose escalation, FL patients received up to six 21-day cycles of obinutuzumab (1000 mg intravenously [IV], Day [D]1, D8, D15 of Cycle [C]1, and D1 of C2-6) and atezolizumab (1200 mg IV, D1 of C2-6) plus pola (1.4 or $1.8 \mathrm{mg} / \mathrm{kg} \mathrm{IV,} \mathrm{D1} \mathrm{of} \mathrm{C1-6).} \mathrm{Subsequently,} \mathrm{patients}$ entered an expansion phase and received obinutuzumab and atezolizumab (same doses) plus pola at the RP2D $(1.8 \mathrm{mg} / \mathrm{kg})$. Patients who achieved at least stable disease at the end of induction (EOI; 6-8 weeks after D1C6) proceeded to obinutuzumab maintenance (1000 mg every 2 months) and atezolizumab (840 mg, D1 and D2 every month) for up to 2 years, or until progressive disease (PD). Unlike the FL cohort, the first seven DLBCL patients entered a safety run-in; once safety criteria were met, the cohort was expanded. All DLBCL patients received up to six 21-day cycles of rituximab $\left(375 \mathrm{mg} / \mathrm{m}^{2} \mathrm{IV}, \mathrm{D} 1 \mathrm{of}\right.$ C1-6), atezolizumab (1200 mg, D1 of C2-6), and pola $(1.8 \mathrm{mg} / \mathrm{kg}$, D1 of C1-6). Patients with at least a partial response (PR) at EOI (6-8 weeks after D1 of C6) received rituximab consolidation $\left(375 \mathrm{mg} / \mathrm{m}^{2}\right.$, D1 every 2 months) and atezolizumab ( $840 \mathrm{mg}, \mathrm{D} 1$ and D2 every month) for up to 8 months, or until PD.

The primary efficacy endpoint was complete response (CR) at EOI by PET-CT (investigator assessed; modified Lugano 2014 criteria) (Cheson et al 2016a, b). Modifications to the standard Lugano criteria were as follows: for the designation of a PR on PET, criteria for CR or PR on CT scan had to be met; if bone marrow (BM) involvement was present at baseline, $\mathrm{CR}$ had to be confirmed with a negative $\mathrm{BM}$ result at end of induction (Cheson et al. 2016a, b).

Overall response rate $(\mathrm{CR}+\mathrm{PR})$ by $\mathrm{PET}-\mathrm{CT}$ was a secondary endpoint. Safety endpoints were the RP2D of pola in combination with G-atezo in FL patients (based on dose-limiting toxicities in $\mathrm{C} 1$ and $\mathrm{C} 2$ ), and the safety/ tolerability of the two regimens in each cohort. The study was conducted in accordance with the Declaration of Helsinki, Good Clinical Practice guidelines, and applicable local laws and regulations. Study materials, including the protocol, were approved by the institutional review boards/ ethics committees at participating centers and patients provided written informed consent.

\section{Statistical analysis}

Safety analyses were conducted in all patients who received $\geq 1$ dose of any study drug (safety population) and are presented by treatment cohort using descriptive statistics. All safety analyses include data collected up to the clinical cut-off date of September 4, 2018.

Efficacy analyses were undertaken in all patients who received $\geq 1$ dose of each component of the G-atezopola or R-atezo-pola combinations (efficacy population). Patients with FL who received polatuzumab vedotin at the RP2D during the dose-escalation phase of the study were pooled for analysis with FL patients who were treated in the expansion phase. Similarly, DLBCL patients from the safety run-in were pooled for analysis with DLBCL patients treated in the expansion phase. Due to early discontinuation of 
atezolizumab for all patients on March 1, 2018, the efficacy population included patients who were not exposed or only partially exposed to atezolizumab. To evaluate the efficacy of induction treatment (primary objective), censoring was applied to the efficacy analyses from March 1, 2018. Response rates are presented using descriptive statistics.

\section{Results}

Between November 9, 2016 and March 1, 2018, 36 patients (R/R FL, $n=13$; R/R DLBCL, $n=23$ ) were enrolled. The clinical cut-off for safety was September 4, 2018. All 13 FL patients were treated and evaluable for safety; $2 / 23$ DLBCL patients did not receive treatment and were not included in the safety population. At the time of data censoring (March 1, 2018), 9/10 FL patients and 10/16 DLBCL patients were evaluable for response. Patient disposition (Fig. 1) and baseline characteristics (Table 1) are presented. Median observation time was 23.3 months (range 3.1-23.7) and 5.7 months (range 0.9-15.4) in the FL and DLBCL cohorts, respectively. On March 1, 2018 (after an interim analysis), study recruitment was stopped permanently due to unexpected safety signals related specifically to G-atezopola, including two fatal adverse events (AEs) (Topp et al. 2020) and limited efficacy. Atezolizumab was discontinued in both cohorts, and due to the lack of evidence supporting extended rituximab monotherapy in DLBCL, rituximab consolidation was ceased in the DLBCL cohort; however, the study remains active for enrolled patients.

At EOI, CR rates in FL patients treated with G-atezo-pola at pola doses of $1.4 \mathrm{mg} / \mathrm{kg}(N=3)$ and $1.8 \mathrm{mg} / \mathrm{kg}(N=7)$ were $33 \%$ (overall response rate [ORR], 33\%) and $14 \%$ (ORR, 57\%), respectively (Table 2). In DLBCL patients who received R-atezo-pola, the $\mathrm{CR}$ rate at $\mathrm{EOI}$ was $13 \%$ (ORR, 25\%).

Safety assessment in FL patients established the RP2D of pola in the G-atezo-pola regimen as $1.8 \mathrm{mg} / \mathrm{kg}$, based on an absence of $\mathrm{C} 1 / \mathrm{C} 2$ dose-limiting toxicities in three patients treated with the $1.4 \mathrm{mg} / \mathrm{kg}$ dose. This is the dose approved for use in combination with rituximab and bendamustine for R/R DLBCL (US FDA 2019). In the FL cohort, 8/13 patients (62\%) experienced a grade 3-5 AE (including two deaths) and 4/13 patients (31\%) developed a serious AE (SAE) (Table 3). One out of 13 patients (8\%) experienced pneumonitis (grade 3 ) and $3 / 13$ patients (23\%) experienced peripheral neuropathy (unresolved in one patient at the time of analysis/death). In DLBCL patients, R-atezo-pola was associated with a lower incidence of grade 3-5 AEs (5/21 patients [24\%]; one death) and SAEs (2/21 patients [10\%]) (Table 3). One out of 21 patients $(5 \%)$ experienced peripheral neuropathy (unresolved at the time of analysis) and no events of pneumonitis were reported in the DLBCL cohort. Across both cohorts, the most common grade 3-5 AEs were hematologic toxicities, although

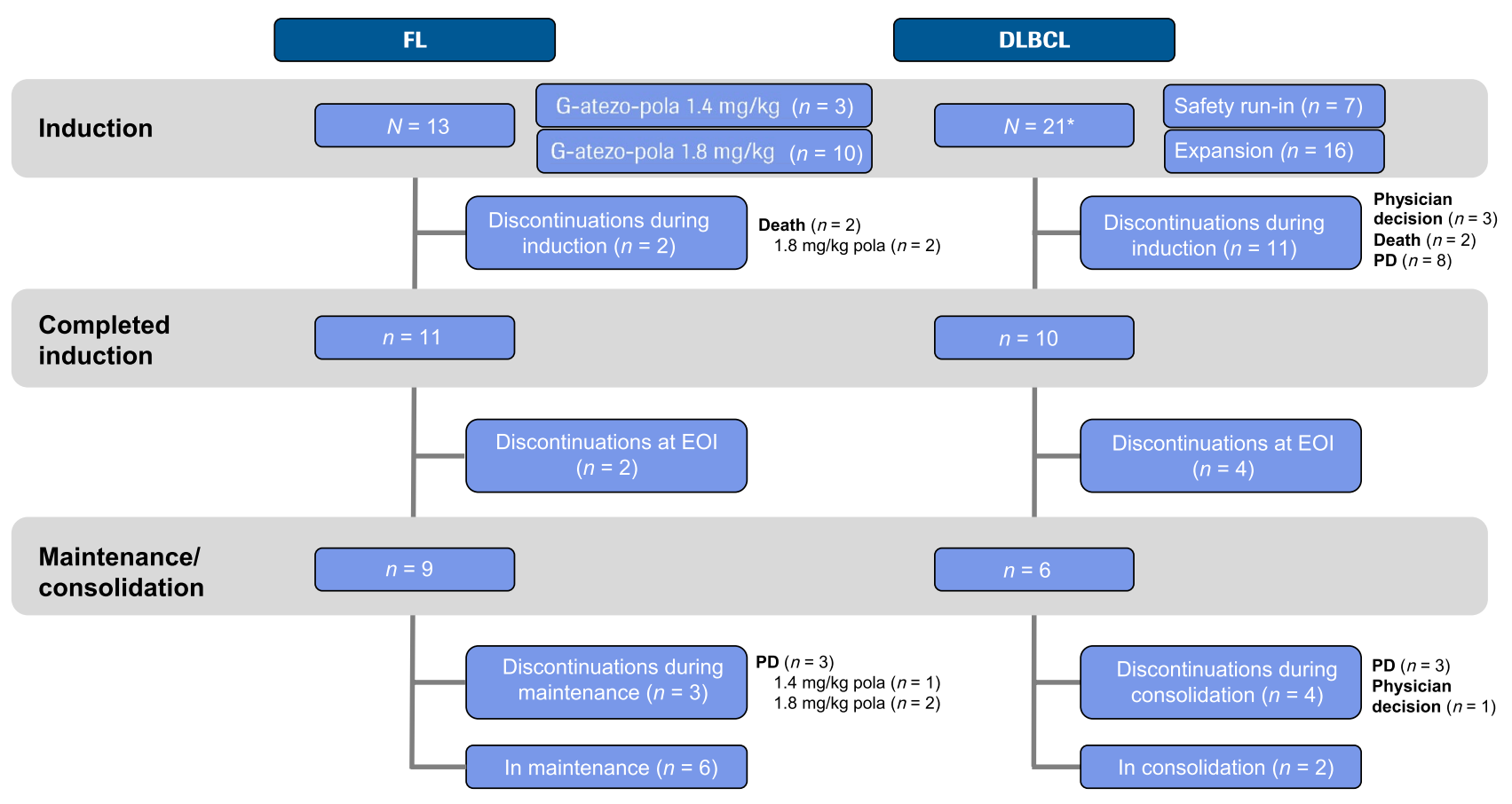

Fig. 1 Patient deposition. *Two additional patients with DLBCL were enrolled but did not receive study treatment and were not included in the safety or efficacy populations. Atezo atezolizumab,
$D L B C L$ diffuse large B-cell lymphoma, EOI end of induction, $F L$ follicular lymphoma, $G$ obinutuzumab, $P D$ progression of disease, pola polatuzumab vedotin, $R$ rituximab, $R / R$ relapsed refractory 
Table 1 Demographics and baseline characteristics of all R/R FL and DLBCL patients (safety population)

\begin{tabular}{|c|c|c|c|}
\hline & \multicolumn{2}{|c|}{$\begin{array}{l}\text { R/R FL } \\
\text { G-atezo-pola }\end{array}$} & \multirow{2}{*}{$\begin{array}{l}\mathrm{R} / \mathrm{R} \text { DLBCL } \\
\text { R-atezo-pola } \\
\text { Pola } \\
1.8 \mathrm{mg} / \mathrm{kg} \\
(N=21)\end{array}$} \\
\hline & $\begin{array}{l}\text { Pola } \\
1.4 \mathrm{mg} / \mathrm{kg} \\
(N=3)\end{array}$ & $\begin{array}{l}\text { Pola } \\
1.8 \mathrm{mg} / \mathrm{kg} \\
(N=10)\end{array}$ & \\
\hline Median age, years (range) & $54(38-65)$ & $58.5(31-71)$ & $68(21-92)$ \\
\hline Male, $n(\%)$ & $3(100)$ & $6(60)$ & $13(62)$ \\
\hline Ann Arbor stage at diagnosis (III-IV), $n(\%)$ & $2(67)$ & $9(90)$ & $18(86)$ \\
\hline \multicolumn{4}{|l|}{ FLIPI score, $n(\%)$} \\
\hline 0-1 (low risk) & $2(67)$ & $3(30)$ & - \\
\hline 2 (intermediate risk) & $1(33)$ & $1(10)$ & - \\
\hline $3-5$ (high risk) & 0 & $6(60)$ & - \\
\hline \multicolumn{4}{|l|}{ IPI score, $n(\%)$} \\
\hline $0-2$ (low risk) & - & - & $7(33)$ \\
\hline 3-5 (intermediate-to-high risk) & - & - & $14(67)$ \\
\hline ECOG PS $0-1, n(\%)$ & $3(100)$ & $10(100)$ & $18(86)$ \\
\hline Bulky disease $(>7.5 \mathrm{~cm}), n(\%)$ & 0 & $6(60)$ & $6(29)$ \\
\hline Bone marrow infiltration, $n(\%)$ & 0 & $2(20)$ & $4(20)$ \\
\hline Extranodal involvement, $n(\%)$ & $1(33)$ & $7(70)$ & $16(76)$ \\
\hline Elevated LDH, $n(\%)$ & 0 & $2(20)$ & $15(71)$ \\
\hline Refractory to last line, $n(\%)$ & $1(33)$ & $8(80)$ & $20(95)$ \\
\hline Relapsed on last line, $n(\%)$ & $2(67)$ & $2(20)$ & $1(5)$ \\
\hline Median prior lines of therapy (range) & $2(1-5)$ & $2(1-4)$ & $2(1-8)$ \\
\hline Prior stem cell transplant, $n(\%)$ & 0 & $1(10)$ & $6(29)$ \\
\hline
\end{tabular}

Atezo atezolizumab, DLBCL diffuse large B-cell lymphoma, ECOG PS Eastern Cooperative Oncology Group performance status, FL follicular lymphoma, FLIPI Follicular Lymphoma International Prognostic Index, $G$ obinutuzumab, IPI International Prognostic Index, $L D H$ lactate dehydrogenase, pola polatuzumab vedotin, $R$ rituximab, $R / R$ relapsed/refractory most events were isolated and reported in one patient only (Table 4).

Both FL patients who died from AEs experienced a new constellation of immune toxicities, which manifested as concomitant severe cutaneous toxicity (dermatitis/ erythema), stomatitis, and ocular AEs (scleritis/ conjunctivitis) refractory to standard corticosteroid and other immunosuppressive treatment; and resembled an autoimmune disease (Stevens-Johnson syndrome) resulting in treatment discontinuation (Topp et al. 2020; Online Supplemental Figure S1). In both cases, patients developed opportunistic infections and other complications related to immunosuppressive therapy. The ultimate cause of death was bronchopulmonary aspergillosis and unknown (no autopsy was performed), respectively. These toxicities were considered by investigators as related specifically to G-atezo-pola. Such presentations were not seen following treatment of DLBCL patients with R-atezo-pola.

\section{Discussion}

Despite prior studies demonstrating the potential of anti-CD20 doublets with atezolizumab or pola in R/R nonHodgkin lymphoma (Morschhauser et al. 2019; Phillips et al. 2016; Palomba et al. 2017) response rates did not appear to improve further in FL and DLBCL patients treated with G-atezo-pola and R-atezo-pola, respectively. In the phase 2 ROMULUS study, CR rates in R/R DLBCL and R/R FL patients treated with R-pola were 16\% (ORR 45\%) and 29\% (ORR 57\%), respectively (Morschhauser et al. 2019). Comparable CR rates were also reported in a phase 1b/2 study investigating G-pola (R/R DLBCL: $28.5 \%$ [ORR 52\%]; R/R FL: 30\% [ORR 78\%]) (Phillips et al. 2016).

In the FL cohort, more patients experienced grade 3-5 AEs with G-atezo-pola compared with patients with DLBCL receiving $\mathrm{R}$-atezo-pola. The potentially better tolerability with R-atezo-pola in patients with DLBCL versus G-atezopola in patients with FL may be due to differences between the anti-CD20 drugs; rituximab may cause less profound B-cell depletion than obinutuzumab (Freeman and Sehn 2018) and, therefore, fewer immune-related AEs (Liudahl 
Table 2 Response at end of induction (investigator assessed $^{\mathrm{a}}$ ) in patients with $\mathrm{R} / \mathrm{R}$ FL and DLBCL

\begin{tabular}{llll}
\hline$n(\%)$ & $\begin{array}{l}\text { R/R FL } \\
\text { G-atezo-pola }\end{array}$ & & $\begin{array}{l}\text { R/R DLBCL } \\
\text { R-atezo-pola }\end{array}$ \\
\cline { 2 - 4 } & $\begin{array}{l}\text { Pola 1.4 mg/kg } \\
(N=3)\end{array}$ & $\begin{array}{l}\text { Pola 1.8 mg/kg } \\
(N=7)\end{array}$ & $\begin{array}{l}\text { Pola 1.8 mg/kg } \\
(N=16)^{\mathrm{c}}\end{array}$ \\
\hline ORR & $1(33)$ & $4(57)$ & $4(25)$ \\
CR & $1(33)$ & $1(14)$ & $2(13)$ \\
PR & 0 & $3(43)$ & $2(13)$ \\
SD & $1(33)$ & $1(14)$ & $1(6)$ \\
PD & $1(33)$ & $1(14)$ & $5(31)$ \\
Not evaluable & 0 & $1(14)$ & $6(38)$ \\
\hline
\end{tabular}

Only patients who received the last dose of study drug in induction before March 1, 2018 or patients with PD or death before March 1, 2018 are included

Atezo atezolizumab, $C R$ complete response, $D o R$ duration of response, $D L B C L$ diffuse large B-cell lymphoma, $E O I$ end of induction, $F L$ follicular lymphoma, $G$ obinutuzumab, $O R R$ overall response rate, $P D$ progression of disease, pola polatuzumab vedotin, $P R$ partial response, $R$ rituximab, $R / R$ relapsed refractory, $S D$ stable disease

${ }^{a}$ Assessed by positron emission tomography-computed tomography according to modified Lugano 2014 response criteria

${ }^{\mathrm{b}}$ To date, a CR has been maintained from EOI in two patients receiving G-atezo-pola; DoR, 21.42 months and 2.79 months, respectively

${ }^{\mathrm{c}}$ To date, a CR has been maintained from EOI in two patients receiving R-atezo-pola; DoR, 10.84 months and 8.97 months, respectively

\begin{tabular}{lll}
\hline & $\begin{array}{l}\text { R/R FL } \\
\text { G-atezo-pola } \\
(N=13)\end{array}$ & $\begin{array}{l}\text { R/R DLBCL } \\
\text { R-atezo-pola } \\
(N=21)\end{array}$ \\
\hline Patients with $\geq 1$ AE, $n(\%)$ & $13(100.0)$ & $17(81.0)$ \\
Grade 3-5 AE, $n(\%)$ & $8(62.0)$ & $5(24.0)$ \\
Grade 5 (fatal) AE, $n(\%)$ & $2(15.0)^{\mathrm{a}}$ & $1(5.0)^{\mathrm{b}}$ \\
Serious AE, $n(\%)$ & $4(31.0)$ & $2(10.0)$ \\
AE leading to any drug discontinuation, $n(\%)$ & $2(15.0)$ & - \\
AE leading to pola dose reduction, $n(\%)$ & $1(8.0)^{\mathrm{c}}$ & - \\
AE leading to any dose interruption or modification, $n(\%)$ & $6(46.0)$ & $3(14.0)$ \\
\hline
\end{tabular}

$A E$ adverse event, atezo atezolizumab, $D L B C L$ diffuse large B-cell lymphoma, $F L$ follicular lymphoma, $G$ obinutuzumab, pola polatuzumab vedotin, $R$ rituximab, $R / R$ relapsed/refractory

${ }^{a}$ Bronchopulmonary aspergillosis $(n=1)$ and unknown $(n=1)$

${ }^{\mathrm{b}}$ Pleural effusion ( $n=1$; a complication of sepsis)

${ }^{c}$ Peripheral neuropathy $(n=1)$ and Coussens 2018) or a lower exposure to atezolizumab and shorter observation time in the DLBCL cohort.

The toxicities that led to fatal outcomes in two patients with FL were considered by investigators as related specifically to G-atezo-pola; however, cutaneous, mucosal, and ocular immune toxicities are already known classrisks for anti-PD-1/PD-L1 therapies, such as atezolizumab (Michot et al. 2016; Brahmer et al. 2018; Collins et al. 2017). The authors of the present study hypothesized potential underlying mechanisms that led to the fatal outcome of these two patients. They suggested that the incidence and severity of these events, known to be associated with checkpoint inhibitors, may be exacerbated in the context of a profound dysregulation of the immune system: obinutuzumab- and polatuzumab vedotin-mediated B-cell suppression, and in particular, regulatory B-cell depletion (Kumar et al. 2015; Goldinger et al. 2016; Zamani et al. 2016; Dai et al. 2014). Furthermore, both patients had received prior treatment with bendamustine, which has been reported to induce regulatory T-cell depletion (Cheson et al. 2016a, b), 
Table 4 Grade 3-5 adverse events reported in $\geq 2 \mathrm{R} / \mathrm{R}$ FL and DLBCL patients

\begin{tabular}{llll}
\hline Preferred term, $n(\%)$ & $\begin{array}{l}\text { R/R FL } \\
\text { G-atezo-pola } \\
(N=13)^{\mathrm{a}}\end{array}$ & $\begin{array}{l}\text { R/R DLBCL } \\
\text { R-atezo-pola } \\
(N=21)^{\mathrm{b}}\end{array}$ \\
\cline { 2 - 4 } & $\begin{array}{l}\text { Pola 1.4 mg } \\
(N=3)\end{array}$ & $\begin{array}{l}\text { Pola 1.8 mg } \\
(N=10)\end{array}$ & $\begin{array}{l}\text { Pola 1.8 mg } \\
(N=21)\end{array}$ \\
\hline Neutropenia & $1(33)$ & $2(20)$ & $2(10)$ \\
Febrile neutropenia & - & $2(15)^{\mathrm{c}}$ & 0 \\
Thrombocytopenia & - & $3(23)$ & 0 \\
Leukopenia & - & $2(15)$ & $1(5)$ \\
Anemia & - & $1(8)^{\mathrm{c}}$ & $1(5)$ \\
Stomatitis & - & $2(15)^{\mathrm{c}}$ & 0 \\
\hline
\end{tabular}

Atezo atezolizumab, DLBCL diffuse large B-cell lymphoma, $F L$ follicular lymphoma, $G$ obinutuzumab, pola polatuzumab vedotin, $R$ rituximab, $R / R$ relapsed/refractory, $S A E$ serious adverse event

${ }^{a}$ Additional isolated grade 3-5 events in patients with R/R FL were bronchopulmonary aspergillosis (SAE; grade 5), pelvic abscess, pneumonitis (SAE; grade 3; resolving), pulmonary embolism, pyrexia (SAE; grade 3; resolved), hypokalemia, sacroiliitis, Guillain-Barré syndrome (suspected; SAE; grade 3; resolving), dermatitis (SAE; grade 3; not resolved), increased lipase, increased gamma-glutamyl transferase, increased C-reactive protein (SAE; grade 3; resolved), increased white blood cell count, increased alanine aminotransferase, and unknown death (SAE; grade 5)

${ }^{\mathrm{b}}$ Additional isolated grade $3-5$ events in patients with $\mathrm{R} / \mathrm{R}$ DLBCL were decreased appetite, diabetes mellitus, abdominal pain (SAE; grade 3; not resolved), fatigue, pneumonia, sepsis (SAE; grade 4; resolved), lethargy (SAE; grade 3; resolved), depression, acute respiratory failure, dyspnea, and pleural effusion (SAE; grade 5)

${ }^{\mathrm{c}}$ One event was classified as an SAE (grade 3; resolving)

potentially contributing to the immune dysregulation. There is insufficient evidence to support an auristatin-derived direct toxicity.

Based on these safety issues, considered as related specifically to the G-atezo-pola triplet, and limited efficacy, no further development of either triplet combination is planned.

Supplementary Information The online version contains supplementary material available at https://doi.org/10.1007/ s00432-021-03847-5.

Acknowledgements The authors would like to thank the patients and their families, and the study investigators, study coordinators, and nurses for the BO29561 study. Editorial support, under the direction of the lead author, was provided by Louise Profit, $\mathrm{PhD}$, of Ashfield MedComms, an Ashfield Health company, and funded by F. Hoffmann-La Roche Ltd.

Author contributions Study design: SC; study conduct: ASK, ELM, GS, ISL, JW, MST, TW, and WK; recruitment and follow-up of patients: AH, ASK, ELM, GS, ISL, JW, MST, TW, UB, and WK; data collection: AH, GS, ISL, UB, and MST; data analysis: GS, ISL, MS, MST, SC, and TN; data interpretation: AH, ASK, ELM, GS, ISL, JW, SC, MS, MST, TN, TW, UB, and WK. All the authors provided their final approval of the manuscript and are accountable for all aspects of the work.

Funding This study was sponsored by F. Hoffmann-La Roche Ltd.

\section{Declarations}

Conflict of interest H.E. reported: research funding, honoraria, or consulting fees from AbbVie, Genentech, Inc., Kite, Gilead Sciences, Takeda, Acerta, AstraZeneca, BeiGene and Juno. M.T. reported: research grants from Amgen, Gilead Sciences, Macrogenics, F. Hoffmann-La Roche Ltd, and Regeneron; consulting fees from Amgen and F. Hoffmann-La Roche Ltd; and advisory board membership for Amgen and Regeneron. T.W. reported: research grants and advisory board membership for F. Hoffmann-La Roche Ltd, Amgen, and Novartis; lecture fees from F. Hoffmann-La Roche Ltd, Novartis, and Celgene; and advisory board membership for Celgene. I.S.L. reported: advisory board membership for Seattle Genetics and Janssen Scientific. J.W. reported: advisory board membership for F. Hoffmann-La Roche Ltd, Celgene, Takeda, Janssen-Cilag, Servier, Amgen, BMS, Incyte, and AbbVie; research funding from F. Hoffmann-La Roche Ltd, GSK/ Novartis, Takeda, Janssen-Cilag, and AbbVie; lecture honoraria from F. Hoffmann-La Roche Ltd, Celgene, Takeda, Janssen-Cilag, and Servier; and conference travel support from Roche. U.B. reported: advisory board membership for F. Hoffmann-La Roche Ltd, Gilead Sciences, Janssen, MSD, and Hexal; and travel grants from Janssen, Amgen, F. Hoffmann-La Roche Ltd, and Gilead Sciences. A.H. reported: research support by F. Hoffmann-La Roche Ltd, Novartis, BMS, Pfizer, and Incyte. E.L.M. reported: advisory board membership for F. Hoffmann-La Roche Ltd, Gilead Sciences, Takeda, JanssenCilag, Amgen, Novartis, AbbVie, and Celgene; lecture honoraria from F. Hoffmann-La Roche Ltd, Janssen-Cilag, Amgen; and conference travel support from F. Hoffmann-La Roche Ltd, Janssen-Cilag, and Amgen. W.K-P. declared no disclosures to report. A.S.K reports that West Virginia University, WV, USA directly received research funding outside the submitted work. T.N., S.C., M.S. and G.S. declared employment at F. Hoffmann-La Roche Ltd/Genentech, Inc. T.N. declared stock ownership at F. Hoffmann-La Roche Ltd/Genentech, Inc.

Data availability Qualified researchers may request access to individual patient level data through the clinical study data request platform (https://vivli.org/). Further details on Roche's criteria for eligible studies are available here: (https://vivli.org/members/ourme mbers/). For further details on Roche's Global Policy on the Sharing of Clinical Information and how to request access to related clinical study documents, see here: (https://www.roche.com/research_and_ development/who_we_are_how_we_work/clinical_trials/our_commi tment_to_data_sharing.htm).

Open Access This article is licensed under a Creative Commons Attribution 4.0 International License, which permits use, sharing, adaptation, distribution and reproduction in any medium or format, as long as you give appropriate credit to the original author(s) and the source, provide a link to the Creative Commons licence, and indicate if changes were made. The images or other third party material in this article are included in the article's Creative Commons licence, unless indicated otherwise in a credit line to the material. If material is not included in the article's Creative Commons licence and your intended use is not permitted by statutory regulation or exceeds the permitted use, you will need to obtain permission directly from the copyright holder. To view a copy of this licence, visit http://creativecommons. org/licenses/by/4.0/. 


\section{References}

Brahmer JR, Lacchetti C, Thompson JA (2018) Management of immune-related adverse events in patients treated with immune checkpoint inhibitor therapy: American Society of Clinical Oncology Clinical Practice Guideline Summary. J Oncol Pract 14:247-249. https://doi.org/10.1200/JCO.2017.77.6385

Cheson BD, Ansell S, Schwartz L et al (2016a) Refinement of the Lugano Classification lymphoma response criteria in the era of immunomodulatory therapy. Blood 128:2489-2496. https://doi. org/10.1182/blood-2016-05-718528

Cheson BD, Brugger W, Damaj G et al (2016b) Optimal use of bendamustine in hematologic disorders: Treatment recommendations from an international consensus panel-an update. Leuk Lymphoma 57:766-782. https://doi.org/10.3109/ 10428194.2015.1099647

Collins LK, Chapman MS, Carter JB, Samie FH (2017) Cutaneous adverse effects of the immune checkpoint inhibitors. Curr Probl Cancer 41:125-128. https://doi.org/10.1016/j.currproblcancer. 2016.12.001

Dai S, Jia R, Zhang X, Fang Q, Huang L (2014) The PD-1/PD-Ls pathway and autoimmune diseases. Cell Immunol 290:72-79. https://doi.org/10.1016/j.cellimm.2014.05.006

Freeman CL, Sehn LH (2018) A tale of two antibodies: obinutuzumab versus rituximab. Br J Haematol 182:29-45. https://doi.org/10. $1111 /$ bjh. 15232

Gao G, Liang X, Jiang J et al (2010) A systematic review and metaanalysis of immunochemotherapy with rituximab for B-cell nonHodgkin's lymphoma. Acta Oncol 49:3-12. https://doi.org/10. 3109/02841860903150502

Goldinger SM, Stieger P, Meier B et al (2016) Cytotoxic cutaneous adverse drug reactions during anti-PD-1 therapy. Clin Cancer Res 22:4023-4029. https://doi.org/10.1158/1078-0432.CCR-15-2872

Kumar V, Abbas A, Aster J (2015) Robbins and cotran pathologic basis of disease, 9th edn. Elsevier, Philadelphia

Liudahl SM, Coussens LM (2018) B cells as biomarkers: predicting immune checkpoint therapy adverse events. J Clin Invest 128:577579. https://doi.org/10.1172/JCI99036

Michot JM, Bigenwald C, Champiat S et al (2016) Immunerelated adverse events with immune checkpoint blockade: a comprehensive review. Eur J Cancer 54:139-148. https://doi.org/ 10.1016/j.ejca.2015.11.016

Morschhauser F, Flinn IW, Advani R et al (2019) Polatuzumab vedotin or pinatuzumab vedotin plus rituximab in patients with relapsed or refractory non-Hodgkin lymphoma: final results from a phase 2 randomised study (ROMULUS). Lancet Haematol 6:e254-e265. https://doi.org/10.1016/S2352-3026(19)30026-2

Palomba ML, Till B, Park SI et al (2017) A phase IB study evaluating the safety and clinical activity of atezolizumab combined with obinutuzumab in patients with relapsed or refractory non-Hodgkin lymphoma (NHL). Hematol Oncol 35:137-138. https://doi.org/10. 1002/hon.2437_126 (Abstract)

Phillips T, Brunvand M, Chen A et al (2016) Polatuzumab vedotin combined with obinutuzumab for patients with relapsed or refractory non-Hodgkin lymphoma: preliminary safety and clinical activity of a phase Ib/II study. Blood 128(Suppl):622. https://doi.org/10.1182/blood.V128.22.622.622 (Abstract)

Ren YR, Jin YD, Zhang ZH, Li L, Wu P (2015) Rituximab treatment strategy for patients with diffuse large B-cell lymphoma after firstline therapy: a systematic review and meta-analysis. Chin Med J (Engl) 128:378-383. https://doi.org/10.4103/0366-6999.150111

Sehn LH, Chua N, Mayer J et al (2016) Obinutuzumab plus bendamustine versus bendamustine monotherapy in patients with rituximab-refractory indolent non-Hodgkin lymphoma (GADOLIN): a randomised, controlled, open-label, multicentre, phase 3 trial. Lancet Oncol 17:1081-1093. https://doi.org/10. 1016/S1470-2045(16)30097-3

TECENTRIQ ${ }^{\circledR}$ (atezolizumab) injection, for intravenous use. Full prescribing information. Genentech, Inc., CA, USA. (2019). https://www.gene.com/download/pdf/tecentriq_prescribing.pdf. Accessed 19 Mar 2021

Topp MS, Duell J, Abajo Guijarro AM et al (2020) Treatmentrefractory T-cell-mediated immune skin toxicities observed with obinutuzumab/rituximab-atezo-pola in two patients with follicular lymphoma. Haematologica 105:e256-260. https://doi.org/10.3324/ haematol.2019.233189

US Food and Drug Administration (2019) FDA approves polatuzumab vedotin-piiq for diffuse large B-cell lymphoma. https://www. fda.gov/drugs/resources-information-approved-drugs/fda-appro ves-polatuzumab-vedotin-piiq-diffuse-large-b-cell-lymphoma. Accessed 19 Mar 2021

Zamani MR, Aslani S, Salmaninejad A, Javan MR, Rezaei N (2016) PD-1/PD-L and autoimmunity: a growing relationship. Cell Immunol 310:27-41. https://doi.org/10.1016/j.cellimm.2016.09. 009

Publisher's Note Springer Nature remains neutral with regard to jurisdictional claims in published maps and institutional affiliations. 